\title{
CAPIVARAS (Hydrochoerus hydrochaeris) E A PRESENÇA DO CARRAPATO (Amblyomma sculptum) NO CAMPUS DA UFSCAR-ARARAS, SÃO PAULO
}

\section{CAPYBARAS (Hydrochoerus hydrochaeris) AND THE PRESENCE OF TICKS (Amblyomma sculptum) AT THE UFSCAR ARARAS CAMPUS, SÃO PAULO}

\author{
Vlamir José Rocha ${ }^{1^{*}}$ \\ Margareth Lumy Sekiama ${ }^{1}$ \\ Daniela Dib Gonçalves ${ }^{2}$ \\ Bruno Rodrigues Sampieri ${ }^{3}$ \\ Gedimar Pereira Barbosa1 \\ Thiago da Costa Dias ${ }^{1}$ \\ Helen Regina Rossi ${ }^{1}$ \\ Piquerobi Freitas Pereira de Souza ${ }^{1}$ \\ 1Universidade Federal de São Carlos, Araras, SP, Brasil. \\ 2Universidade Paranaense, Umuarama, PR, Brasil. \\ 3Universidade Estadual Paulista, Rio Claro, SP, Brasil \\ *Autor para correspondência - vlamir@cca.ufscar.br
}

\section{Resumo}

Capivaras são territoriais e vivem em grupos sociais, ocorrendo comumente em áreas antropizadas. Em algumas regiões do Brasil, estes animais apresentam infestação por carrapatos do gênero Amblyomma, que por sua vez são considerados como principal vetor biológico e reservatório natural da bactéria Rickettsia rickettsii, agente causador da Febre Maculosa Brasileira. Na UFSCar campus Araras, a interação entre capivaras, carrapatos e comunidade acadêmica é frequente, ocorrendo principalmente durante a estação seca. Este estudo teve como objetivo realizar um censo populacional das capivaras que ocupam o campus, identificando sua área de uso e se estes roedores se encontravam infestados por carrapatos. Realizou-se observação direta das capivaras, captura-marcação e recaptura com uso de um brete, e também coleta dos carrapatos nas capivaras e no ambiente. Registrou-se um total de 56 capivaras, com densidade de 1,50 ind./ha. Foram capturados 24 animais, sendo $25 \%$ machos e $75 \%$ fêmeas, dos quais $25 \%$ eram adultos, $45,8 \%$ jovens e $29,2 \%$ filhotes. A espécie de carrapato amplamente encontrada infestando tanto os animais como o ambiente foi A. sculptum. Conclui-se que o grupo de capivaras apresentou um número elevado de indivíduos por causa da abundância de recursos e ausência de predadores, e também com grande infestação por carrapatos.

Palavras-chave: capivaras; censo; comunidade acadêmica; manejo; saúde pública.

\section{Abstract}

Capybaras are territorial animals that live in groups social occurring even in anthropized areas. In some 
regions of Brazil, these animals have shown high rates of tick infestation from the genus Amblyomma, which can be considered as a main biological vector and natural reservoir of the bacteria Rickettsia rickettsii, an agent that causes Brazilian Spotted Fever. At Federal University of São Carlos, Araras campus, the interaction between capybaras, ticks and the community is frequent, occurring especially during the dry season. This study aimed to carry out a population census of capybaras that occurred on campus, identifying their home-range and verifying if these rodents were infested by ticks. The Applied methods included direct observation of capybara, capture-mark-recapture technique using a trap and also collection of ticks from the capybaras and the environment. The census recorded a maximum number of 56 capybaras, with density of 1.50 individuals/ha. The trap captures accounted for 24 animals, $25 \%$ males and $75 \%$ females, in which $25 \%$ were adult animals, $45.8 \%$ juveniles and $29.2 \%$ infants. The tick species most found both infesting animals and environment was A. sculptum. In conclusion, the group of capybaras studied showed a high number of individuals of resources and due to the presence the absence of predators, and given the high ticks infestation.

Keywords: capybaras; census; academic community; management; public health.

Recebido em: 19 de dezembro de 2016

Aceito em 01 de setembro de 2017

\section{Introdução}

Capivaras (Hydrochoerus hydrochaeris Linnaeus, 1766) são animais que vivem em grupos que podem chegar a mais de 50 indivíduos ${ }^{(1)}$ com forte organização social e estrutura hierárquica ${ }^{(2)}$. A ocorrência destes mamíferos está sempre associada a cursos de água como rios e lagos artificiais ou naturais que fornecem proteção, possibilidade de termorregulação e local propício para a cópula ${ }^{(3,4)}$. Possuem áreas de vida que podem ter tamanhos variados conforme a disponibilidade de recursos e o tamanho do grupo, mas que raramente ultrapassam $500 \mathrm{~m}$ de distância dos corpos d'água ${ }^{(4-10)}$.

Com ninhadas que vão de um a oito filhotes e gestação com duração média de 150 dias, a capivara possui taxas elevadas de fecundidade e fertilidade ${ }^{(11)}$, e o desequilíbrio ambiental, a substituição da mata nativa por culturas agrícolas e pastagens e a ausência de predadores naturais vêm contribuindo consideravelmente para o aumento das populações destes animais em algumas regiões do Brasil, com destaque para o estado de São Paulo ${ }^{(12-16)}$, inclusive com o registro destes roedores em parques urbanos ${ }^{(17-}$ 19).

Esta situação coloca estes animais cada vez mais em contato com o homem ${ }^{(20)}$, resultando em problemas como danos e prejuízos causados em culturas agrícolas ${ }^{(13,21,22)}$, animais em rodovias que provocam colisão com veículos, reduzindo a segurança nas estradas ${ }^{(23,24)}$ e, o mais preocupante, o número elevado de capivaras podem contribuir para o crescimento da população de carrapatos, consequentemente aumentando a possibilidade de transmissão de doenças associadas a este ectoparasita ${ }^{(25-30)}$.

As capivaras são consideradas um dos principais hospedeiros de carrapatos Amblyomma spp. na América do Sul $^{(26)}$ e no interior do estado de São Paulo. Perez et al. ${ }^{(31)}$ registraram que a capivara, entre outros mamíferos analisados, foi a que apresentou a maior quantidade de carrapatos adultos do gênero Amblyomma, principalmente da espécie Amblyomma cajennense (sensu lato) (Fabricius 1787). 
Os carrapatos pertencentes a este gênero são considerados como principal vetor biológico e reservatório natural da bactéria Rickettsia rickettsii, causadora da Febre Maculosa Brasileira (FMB), sendo no estado de São Paulo a espécie Amblyomma sculptum (Berlese, 1988) de ampla ocorrência em áreas periurbanas e rurais, atuando como reservatório e vetor do agente da $\mathrm{FMB}^{(27,29,30,32-36)}$.

Além disso, tem-se reconhecido o papel da capivara como amplificadora dessa bactéria, pois, quando capivaras ainda não infectadas são picadas por carrapatos portadores da Rickettsia, desenvolvem assintomaticamente a doença e contaminam um número elevado de carrapatos não infectados que entram em contato com esse roedor durante o período de bacteremia ${ }^{(29,37-39)}$.

No campus da UFSCAR-Araras as interações entre capivaras, carrapatos e comunidade acadêmica são frequentes, ocorrendo principalmente durante a estação seca, entre abril e setembro, período em que a oferta de alimentos no ambiente natural é reduzida e as capivaras tendem a ampliar suas áreas de deslocamento junto aos plantios experimentais de cana-de-açúcar para se alimentarem principalmente à noite ou agruparem-se próximas às margens dos lagos artificiais pela manhã e final da tarde, locais que ainda mantêm uma vegetação mais verde e adequada ao forrageio desses animais (Obs. Pess.).

Durante este período, também é observado o aumento da presença de carrapatos nas áreas naturais do campus, principalmente de larvas e ninfas ("micuins") que nos períodos de outono/inverno exibem o comportamento de busca ativa por hospedeiro, subindo nas pontas da vegetação após passarem por período de diapausa no solo logo após a eclosão dos ovos durante a estação chuvosa ${ }^{(40)}$. Dessa forma, aumentam consideravelmente os riscos em relação à transmissão da FMB, por ser esta uma região endêmica desta enfermidade ${ }^{(41-44)}$.

Embora essa questão seja de grande importância para a saúde pública, nenhum trabalho foi realizado com capivaras no município de Araras-SP, portanto, entender sobre a dinâmica populacional destes roedores e a presença de carrapatos nos ambientes alterados pode auxiliar no entendimento desta epidemiologia, bem como se propor formas de manejo da capivara e o controle da FMB.

Diante deste cenário, este estudo teve como objetivos realizar um censo populacional, identificar a área de uso do grupo e detectar a presença de carrapatos nas capivaras (Hydrochoerus hydrochaeris) do campus da UFSCar - Araras.

\section{Material e Métodos}

A Universidade Federal de São Carlos (UFSCar), campus de Araras, está localizada no município de

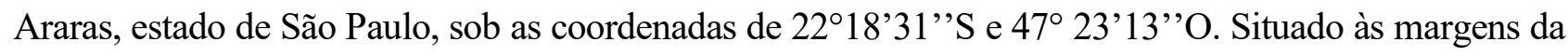
rodovia Anhanguera, no km 174, o campus possui uma área de 230 ha, sendo que uma pequena parte é ocupada por edificações (salas de aulas, laboratórios, administração, etc.), aproximadamente 130 ha são ocupados por áreas experimentais de cana-de-açúcar, e 12,7 ha são constituídos por um fragmento florestal alterado, caracterizado como floresta estacional semidecidual e 23,7 ha de espelho de água distribuídos em três lagos de diferentes tamanhos, 3,7 ha correspondem à área de pasto composto principalmente de capim colonião (Panicum maximum), restante são de áreas em processo de restauração florestal de diferentes idades às margens dos lagos (Figura 1). 

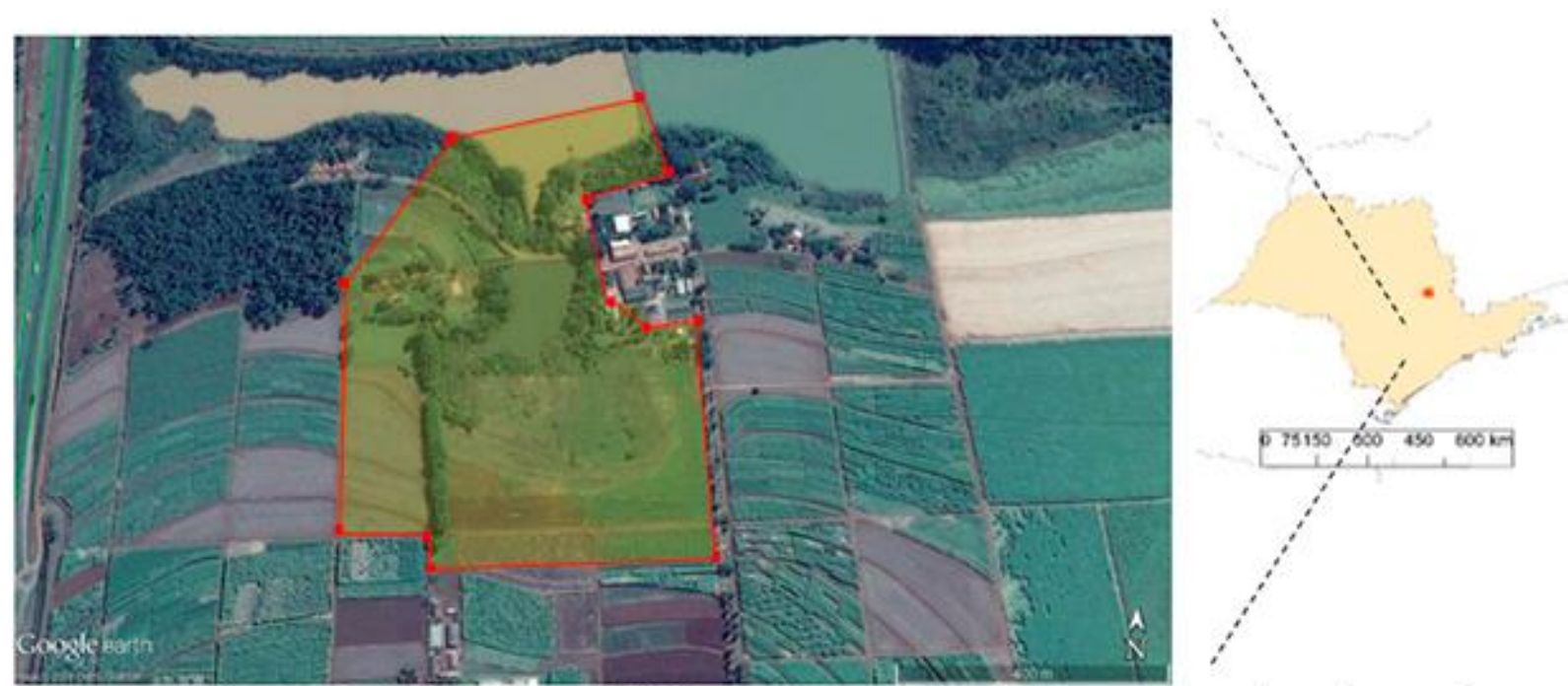

Figura 1. Vista parcial do campus UFSCar - Araras, com destaque para a área de uso das capivaras registrada com os pontos mais externos do poligono (método do MPC). Fonte: Google Earth, data: Junho 2013.

O estudo se concentrou em um grupo de capivaras que frequentava o lago menor, com 1,7 ha alimentados por cinco nascentes. No seu entorno existe uma vegetação herbácea com predomínio de capim marmelada (Brachiaria plantaginea), uma vez que no passado já houve criação de gado na área. Desde 2008, a área destinada à APP (área de preservação permanente) em torno do lago vem sendo enriquecida através de processo de restauração com espécies nativas, que formam atualmente arvoretas e até um pequeno bosque na margem esquerda do lago. Toda a área de APP é rodeada por plantios experimentais de cana-de-açúcar e por um pasto com capim colonião (Panicum maximum cv. Mombassa), onde as capivaras forrageiam.

O clima da região apresenta duas estações bem definidas, uma chuvosa de outubro a março e outra seca de abril a setembro. Os dados históricos de pluviosidade no local registram média de $230 \mathrm{~mm}$ para os meses mais chuvosos, e uma média de $36 \mathrm{~mm}$ para os meses mais secos. Segundo o sistema de classificação de Köppen, o clima é do tipo Cwa, mesotérmico com verões quentes e úmidos e invernos secos, com temperaturas médias acima de $22{ }^{\circ} \mathrm{C}$ no mês mais quente e acima de $18{ }^{\circ} \mathrm{C}$ no mês mais frio $^{(45,46)}$.

Os dados em campo foram coletados entre abril e dezembro de 2013, mas as contagens dos indivíduos ocorreram no período de abril a setembro de 2013 através do método de observação direta, independentemente da faixa etária e sexo e com auxílio de binóculo 8x40. Os locais utilizados pelas capivaras foram percorridos a pé uma vez por semana, durante o período da manhã, por volta das 7:30h até cerca de 10:00h, ou à tarde, por volta das 17:00h até cerca de 19:30h.

A observação direta é a metodologia mais utilizada para estimar os índices de abundância dos grupos de capivaras $^{(1,4,6,47-49)}$ em função das características morfológicas, comportamentais e do tipo de habitat desse animal. Mesmo que essa metodologia não determine a densidade absoluta da população por partir da contagem do tamanho mínimo imediato do grupo de animais ${ }^{(50)}$, há uma correlação significativa entre esse índice de abundância e a densidade real da população ${ }^{(51-54)}$.

Para a determinação da densidade populacional de capivaras, foi considerado o número máximo de indivíduos (n) observados durante a contagem dividido pela área de uso do grupo. Durante o percurso 
também foram feitos alguns registros das pegadas e fezes que se encontravam mais distantes do lago, como método para verificar a distância máxima de deslocamento dos animais em linha reta a partir do lago e também para calcular a área de uso do grupo através do Método do Mínimo Polígono Convexo (MPC) considerando $100 \%$ dos pontos mais externos registrados no polígono ${ }^{(55-57)}$ (Figura 1). As medidas e plotagens dos pontos foram feitas com o programa Google Earth Pro 7.1.5.1557.

Paralelamente, foi construído em local sombreado, próximo à margem do lago, um brete semelhante ao descrito por Pereira \& Eston ${ }^{17}$, com $6 \mathrm{~m}$ de largura por $5 \mathrm{~m}$ de comprimento, e 1,50 $\mathrm{m}$ de altura, na área mais utilizada pelo grupo. Na construção do brete foi utilizado tela de arame galvanizado fio 8, malha 10 $\mathrm{cm}$, oito mourões de concreto para fixação da tela e porta do tipo guilhotina, acionada pelo próprio animal quando este pisava no sistema de desarme do tipo "trampa". No fundo do brete foi posicionado um cocho onde eram colocados semanalmente milho, sal grosso e folhas de bananeira como forma de atrair os animais para o interior da armadilha. Uma lona plástica preta foi colocada ao redor do brete para diminuir o estresse e evitar que os animais se jogassem contra a tela no momento da captura.

Após um período de 5 meses de habituação com o brete, iniciaram-se as campanhas de capturas, sendo uma em setembro, duas em novembro e a última campanha em dezembro de 2013. Todos os animais capturados foram contidos fisicamente com o auxílio de puçá e logo após foi colocado um pano na cabeça do animal de forma que cobrisse seus olhos como forma de mantê-los calmos. Posteriormente, foram sexados e classificados em adultos (acima de $30 \mathrm{Kg}$ ), jovens (de 10 a $30 \mathrm{Kg}$ ) e filhotes (abaixo de $10 \mathrm{Kg}$ ) conforme os critérios de Ojasti ${ }^{(3)}$.

Realizou-se também estimativa populacional das capivaras com o uso do software livre Capture ${ }^{(58-60)}$ através do método de captura-marcação-recaptura (CMR) para populações fechadas. Método este que, segundo Fernandez ${ }^{(61)}$, leva em conta os parâmetros de população presentes no momento, não considerando alterações como nascimentos, mortes, emigrações ou imigrações, o que se adequava ao período curto das capturas realizadas (final de setembro a início de dezembro de 2013).

Os animais foram marcados em uma das orelhas inicialmente com brincos metálicos numerados de tamanho $5 \mathrm{~cm} \times 1,5 \mathrm{~cm}$, com formato retangular e aplicação manual e posteriormente com brincos plásticos numerados de tamanho 3,6cm x $1 \mathrm{~cm}$ de mesmo formato, mas aplicados com o uso de alicates específicos para esta finalidade. Após o manuseio, as capivaras foram liberadas no mesmo local.

Realizou-se uma avaliação das capivaras em relação a presença de carrapatos e, aleatoriamente, coletouse manualmente de 1 a 10 ectoparasitas diretamente dos hospedeiros. Paralelamente, foi realizado ao redor do lago, onde as capivaras forrageavam frequentemente, uma coleta de carrapatos adultos pelo método de arrasto com o uso de um tecido branco de $1 \mathrm{~m}^{2(62)}$.

Os carrapatos coletados foram colocados em tubos Falcon e congelados em freezer a $-18^{\circ} \mathrm{C}$, enquanto os que foram coletados do ambiente foram mantidos vivos em potes coletores para posterior fixação e aplicação da técnica de microscopia eletrônica de varredura. Em seguida, as amostras foram enviadas ao laboratório do grupo BCSTM (Brazilian Central of Studies on Ticks Morphology), do Departamento de Biologia da UNESP - campus Rio Claro, para confirmação da espécie através de caracteres morfológicos externos, segundo metodologia proposta por Flechtmann ${ }^{(63)}$, Barros-Battesti et al. ${ }^{(64)}$ e Nava et al. ${ }^{(36)}$, utilizando-se das seguintes características:

a) macho - dentículos do hipostômio no padrão 3/3 distribuídos em seis linhas (Figura 2A); um par de espinhos coxais nas coxas I, sendo o mais externo maior e o mais interno menor (Figuras $2 \mathrm{~B}$ e C); abertura 
genital entre as coxas II com forma de U (Figura 2B); b) fêmea - dentículos do hipostômio no padrão 3/3 distribuídos em 7 linhas (Figura 2D1); um par de espinhos coxais nas coxas I, sendo o mais externo maior e pontiagudo e o mais interno menor com extremidade arredondada (Figuras 2D e E); abertura genital entre as coxas II e III, estreita com forma de U e um espinho coxal nas coxas IV, curto e arredondado (Figura 2F).

Os carrapatos coletados foram fixados em Etanol 70\% por no mínimo 24 horas, após anestesia por choque térmico em freezer. Na sequência o material foi desidratado em concentrações crescentes de acetona (80, 85, 90, 95 e 100\%) em banhos de cinco minutos em cada concentração, sendo que na acetona $100 \%$ realizaram-se dois banhos. Após a dessecação em Critical Point Drying, os exemplares foram colados com fita adesiva dupla face em suportes de alumínio, metalizados com ouro em "Sputtering", examinados e fotografados em microscópio eletrônico de varredura Hitachi TM 3000, no laboratório de Microscopia Eletrônica da UNESP, Rio Claro, SP.
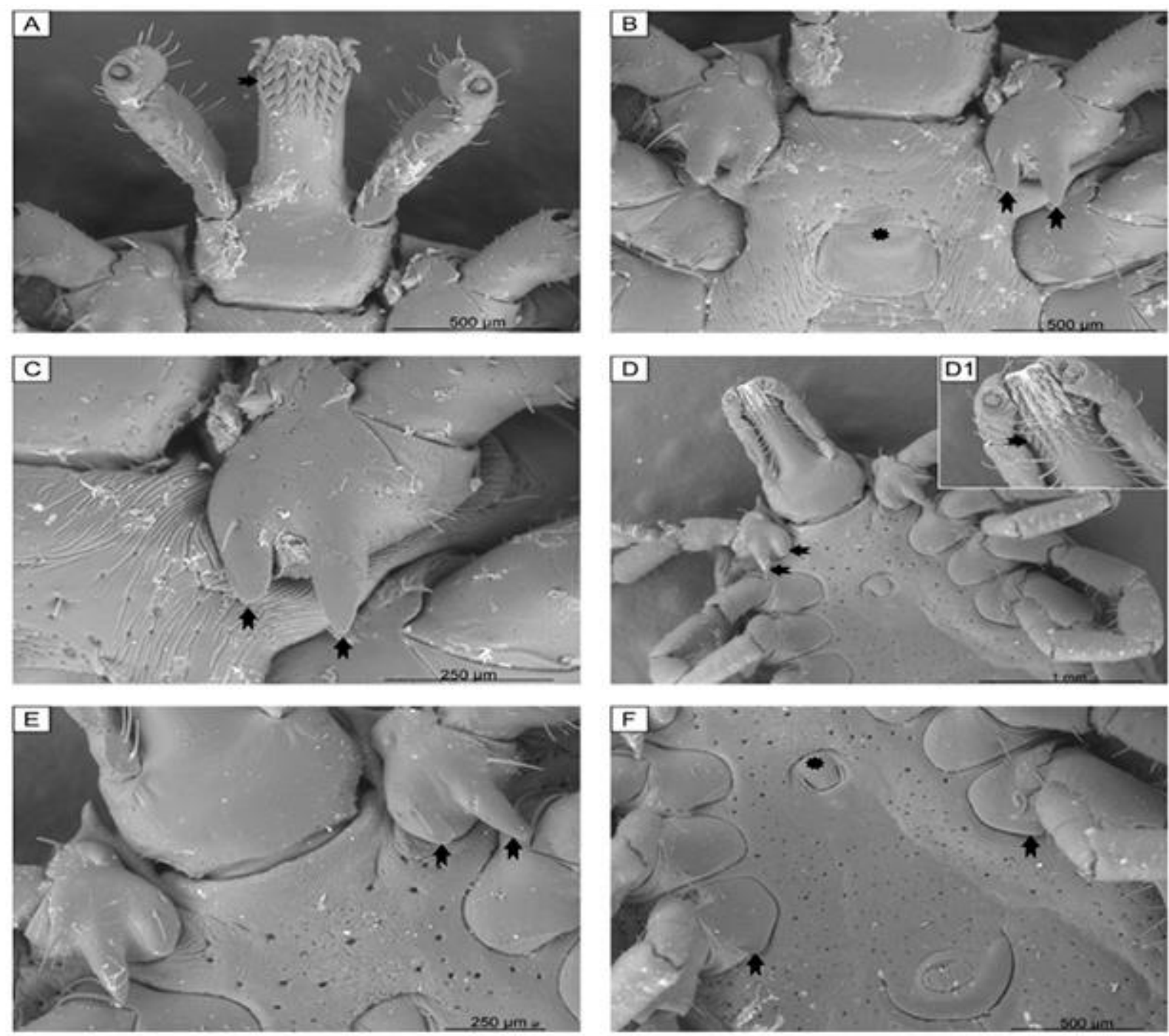

Figura 2. Identificação de adultos de Amblyomma sculptum através de caracteres morfológicos externos (imagem de microscopia de varredura). 


\section{Resultados}

O número máximo de capivaras registradas foi de 56 indivíduos para o mês de junho e a densidade populacional foi de 1,50 ind/ ha. A média de indivíduos observados por semana foi de 9,64, número este bem abaixo do número máximo registrado. Paralelamente, foram registrados mais dois grupos em áreas marginais ao campus, ocupando os outros dois lagos, porém estes não tiveram os indivíduos contabilizados por não serem o foco dos resultados.

No brete foram capturados um total de 24 animais, sendo que, três foram recapturas, incluindo o macho alfa do grupo. Seis indivíduos eram machos (25\%) e 18 fêmeas (75\%) uma proporção de 3:1, e as classes etárias registradas foram de seis adultos (25\%), 11 jovens $(45,8 \%)$ e sete filhotes $(29,2 \%)$.

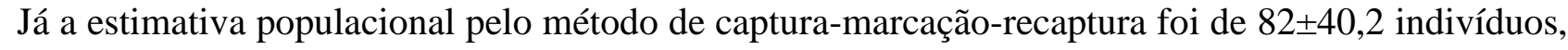
o que representaria uma densidade máxima de 2, 20 ind/ ha $\pm 1,08$.

A área de uso do grupo foi estimada em 37,14 ha (Figura 1), a partir de 12 registros mais externos de pegadas/fezes. A área de vida das capivaras foi delimitada em sua porção sul e leste por uma cerca. A distância máxima de deslocamento em linha reta a partir do lago foi de 430 metros.

Duas espécies de carrapatos foram identificadas parasitando as capivaras: Rhipicephalus sanguineus sl (sensu lato) apenas uma fêmea e Amblyomma sculptum, cerca de 70 adultos (entre machos e fêmeas) além de grande quantidade de larvas e ninfas.

Já na coleta realizada nas redondezas do lago, na serapilheira, foi observada vasta presença de larvas e ninfas sobre a vegetação rasteira, bem como foram coletados cerca de 50 indivíduos adultos de $A$. sculptum em aproximadamente 15 minutos de aplicação do método de arrasto.

\section{Discussão}

O número máximo de capivaras registrado $(\mathrm{n}=56)$ para o grupo estudado no campus da UFSCar Araras corrobora com outros trabalhos obtidos em ambientes semelhantes com áreas antrópicas e de extensas áreas agrícolas e pastagens ${ }^{(13-15,17,22,50)}$. Nesses locais, os grupos podem chegar a conter mais de 50 animais, número elevado se comparado a ambientes com menor grau de perturbação e interferência antrópica, como mostram os estudos realizados no Pantanal Brasileiro, com média de animais por grupo variando de 3,6 a 9,48 indivíduos ${ }^{(6,47,50,65,66)}$, e nos Lhanos Venezuelanos, com média de 10,9 a 14 indivíduos por grupo ${ }^{(1-4)}$.

Ojasti ${ }^{(3)}$ menciona que esses grupos numerosos são formados durante a estação seca, quando as capivaras se agregam próximo a corpos d'água. $\mathrm{Na}$ área do campus é provável que exista uma tendência do grupo se manter numeroso ao longo do ano todo, já que é grande a oferta de alimento disponível, principalmente de cana-de-açúcar, inclusive com danos a esta cultura causado pelas capivaras. Segundo Ferraz et al. ${ }^{(21)}$ e Verdade \& Ferraz ${ }^{(13)}$, nestas áreas alteradas, as fontes de alimentos disponíveis e a extinção local de grandes predadores são dois dos principais fatores que levam ao aparecimento desses grandes grupos, inclusive em matriz agrícola ${ }^{(21,22)}$, uma vez que as 
capivaras são herbívoros generalistas e oportunistas ${ }^{(67)}$.

Com relação à densidade populacional, os valores obtidos através da contagem direta dos animais ( $\mathrm{n}=1,5 \mathrm{ind} / \mathrm{ha}$ ) ultrapassam os encontrados por Verdade e Ferraz ${ }^{(13)}$, para o campus da Esalq-USP no município de Piracicaba (1,24 ind/ha); por Vargas et al. ${ }^{(68)}$ para o município de Pirassununga (0,82 ind/ha); e por Silva et al. ${ }^{(69)}$ para a região de Londrina estado do Paraná (1,18 ind/ha). Por outro lado, em área com menor influência antrópica, as densidades são bem menores como as encontradas por Alho et al. ${ }^{(70)}$ no Pantanal Brasileiro com 0,01 a 0,69 ind/ha, e as densidades registradas por Eisenberg et al. (1979) apud Ferraz et al. ${ }^{(50)}$ e Herrera $(1986)^{(71)}$ apud Ferraz et al. ${ }^{(50)}$ nos Lhanos Venezuelanos, com 0.10 a 1.3 ind/ha.

Foi possível verificar também que a população estimada pelo método de captura-marcação-recaptura $(82 \pm 40)$ foi maior que a população registrada através do método de observação direta $(\mathrm{n}=56)$. Tal variação pode estar relacionada com a baixa taxa de recapturas $(n=3)$, em função do elevado número de indivíduos que constituem o grupo estudado, refletindo diretamente no estimador estatístico.

Quanto à marcação por brincos metálicos e plásticos, apesar de Salas et al. ${ }^{(72)}$, em seu trabalho na Venezuela, mencionarem que brincos plásticos possa ser o melhor método de marcação para estudos de longo prazo, os autores não informam o tamanho e tipo de formato do brinco, e no presente estudo os brincos retangulares plásticos e metálicos aplicados nas orelhas das capivaras saíram após alguns dias.

Pois tanto os animais observados posteriormente, bem como os animais recapturados estavam sem os brincos, com evidência de cicatriz e até mesmo rasgos na orelha que havia sido marcada. Pressupõese que a perda dos brincos possa estar relacionada ao fato dos animais enroscarem as orelhas em cercas, galhos e até mesmo um animal por curiosidade acabar retirando do outro com o uso dos dentes, porém, mais investigações se mostram necessárias. Certamente, a utilização da técnica de microchipagem $^{(68)}$ poderia resolver este tipo de situação, tornando permanente a marcação nos animais, todavia esta é uma técnica que necessita de recapturas para a identificação do indivíduo.

Sobre o sexo e faixa etária dos animais capturados, o maior número de fêmeas e jovens, comparado ao número de machos adultos, pode ser compreendido pela estrutura social destes animais, que é formada por um macho dominante, várias fêmeas adultas, indivíduos jovens e filhotes, além de poucos machos adultos submissos, podendo ser classificado como do tipo harém ${ }^{(3,68)}$. A proporção de três fêmeas para cada macho (3:1) relatada no estudo foi igual à média obtida por Alho \& Rondon $^{(65)}$ no Pantanal, e pouco maior que a obtida por Herrera \& Macdonald ${ }^{(4)}$ na Venezuela (2:1). Portanto, é possível dizer que a razão sexual encontrada nos grupos de capivaras na área do estudo caracterizada como ambiente antrópico é semelhante às encontradas em ambientes naturais ${ }^{(18)}$.

Neste estudo, a área de vida das capivaras (37,14 ha), aproxima-se das áreas registradas por Verdade \& Ferraz ${ }^{(13)}$, com 40,8 hectares no campus da Esalq-USP em Piracicaba, por Corriale et al. ${ }^{(7)}$, que relatam 27,6 ha em uma antiga fazenda de gado na Argentina e por Perea \& Ruíz ${ }^{(5)}$ que registraram 56 ha na Colômbia, sendo consideravelmente maior que as áreas encontradas nos Lhanos Venezuelanos, com tamanho variando de 5 a 16,3 ha $^{(4,7)}$, e Pantanal Brasileiro ${ }^{(65)}$, com média de 12 ha por grupo. Todavia, também no Pantanal Brasileiro ${ }^{(6)}$ registrou-se uma área superior com 200 ha.

Modificações na área de vida de representantes da ordem Caviomorpha estão diretamente relacionadas a fatores como o nível de predação, densidade populacional, e disponibilidade de 
recursos alimentares ${ }^{(73)}$. Para capivaras, destaca-se também a presença de corpos d'água, recurso fundamental para realização das atividades vitais da espécie como proteção, termorregulação e cópula ${ }^{(3,4)}$.

Herrera \& Macdonald ${ }^{(4)}$ descrevem correlação positiva entre área de vida e tamanho do grupo. No entanto, Corriale et al. ${ }^{(7)}$ não encontraram correlação positiva entre o tamanho dos grupos de capivaras e sua área de vida, sugerindo que abundância e disponibilidade de recursos alimentares são os principais fatores limitantes na determinação do tamanho da área de vida do grupo e não o tamanho do grupo, situação que parece se aplicar a este estudo.

A busca por recursos alimentares dentro da área de vida definiu também o máximo deslocamento das capivaras (aproximadamente $430 \mathrm{~m}$ em linha reta) a partir do lago. Esta distância engloba as áreas de plantações de cana-de-açúcar, sendo este o principal recurso consumido pelos animais. Essa distância é semelhante da registrada por Almeida \& Biondi ${ }^{(19)}$, em que os animais se deslocaram 485 metros a partir do lago que ocupavam em um parque urbano na cidade de Curitiba, PR.

Quanto à presença de carrapatos, o predomínio de A. sculptum ectoparasitando as capivaras corrobora com o descrito por Perez et al. ${ }^{(31)}$, que verificaram que $80 \%$ dos carrapatos encontrados em capivaras na área da Esalq/USP no município de Piracicaba pertenciam a essa espécie. Queirogas et al. ${ }^{(74)}$ também registraram 77,3\% desta espécie, enquanto que 22,7\% eram de Amblyomma dubitatum em capivaras no município de Uberlândia, MG.

A alta incidência de A. sculptum pode ser considerada preocupante, uma vez que essa espécie é descrita como um dos principais vetores da Rickettsia rickettsii, bactéria responsável pela transmissão da febre maculosa em seres humanos ${ }^{(33)}$.

O registro de uma fêmea de $R$. sanguineus sl encontrado em uma das capivaras pode estar correlacionado com a presença de cães na área do campus, os quais frequentemente interagiam agonisticamente com as capivaras. Todavia, este fato amplifica a preocupação relativa à saúde pública, uma vez que essa espécie de carrapato possui potencial para transmitir o agente da FMB, por atuar como vetor e ser considerado praga urbana, infestando principalmente cães e se adaptando facilmente a ambientes domiciliares e peridomiciliares ${ }^{(75-77)}$.

Da mesma forma, enfatiza-se a necessidade de novos estudos, confirmando a possível contaminação das capivaras do campus por $R$. rickettsii, bem como dos carrapatos, uma vez que a capacidade de dispersão destes artrópodes é muito ampla devido aos altos índices de parasitismo por imaturos (larvas e ninfas) em aves passeriformes, inclusive migratórias ${ }^{(78)}$, o que pode facilitar a propagação da ocorrência de FMB e de outros agentes infecciosos de outras zoonoses.

A partir dos resultados obtidos foi possível realizar campanhas de esclarecimentos no campus, além da confecção de placas alertando a comunidade acadêmica e seu entorno sobre a presença de carrapatos e os riscos de transmissão da febre maculosa.

\section{Conclusão}

Pode-se afirmar que a população de capivaras do campus da UFSCar - Araras apresenta-se muito 
numerosa, uma vez que essa área fornece os recursos necessários para proliferação da espécie, como corpos d'água e alimentação em abundância, principalmente proveniente do plantio de cana-deaçúcar. O elevado número destes roedores também pode aumentar os riscos à saúde pública, uma vez que propicia um efeito cascata, aumentando a população de carrapatos que acaba por aumentar a interação com a comunidade acadêmica trazendo risco de transmissão de zoonoses, como a febre maculosa. Considerando esses riscos, faz-se necessária a realização de novos estudos que visem monitorar e realizar o correto manejo da população de capivaras e dos carrapatos do campus.

\section{Aspectos Éticos}

Este projeto foi aprovado pelo Instituto Chico Mendes de Conservação da Biodiversidade - ICMBio sob o protocolo n ${ }^{\text {o. }}$ 37227-1 e pelo Comitê de Ética em Pesquisa Envolvendo Experimentação Animal - CEPEEA) da Universidade Paranaense (UNIPAR) sob o protocolo $\mathrm{n}^{\text {o. }}$ 26226/2014.

\section{Agradecimentos}

Agradecimentos à Proex (Pró-Reitoria de Extensão da Universidade Federal de São Carlos) pelo apoio financeiro e bolsas concedidas.

\section{Referências}

1. Macdonald DW. Dwindling resources and the social behaviour of capybara (Hydrochoerus hydrochaeris) (Mammalia). Journal of Zoology. 1981;194(3):371-391.

2. Herrera EA, Salas V, Congdon ER, Corriale MJ, Tang-Martínez Z. Capybara social structure and dispersal patterns: variations on a theme. Journal of Mammalogy. 2011;92(1):12-20.

3. Ojasti J. Estudio biologico del chigüire o capibara. Caracas: Fondo Nacional de Investigaciones Agropecuarias (FONAIAP)/Editorial Sucre; 1973.

4. Herrera EA, Macdonald DW. Resource utilization and territoriality in group-living capybaras. Journal of Animal Ecology. 1989;58(2):667-679.

5. Perea J, Ruíz S. Organización social y hábitos territoriales del cacó [dissertation]. Santa Fé de Bogotá: Universidad Nacional; 1977.

6. Schaller GB, Crawshaw PG. Social organization in a capybara population. Säugetierkundliche Mitteilungen. 1981;29:3-16.

7. Corriale MJ, Muscheto E, Herrera EA. Influence of group sizes and food resources in home-range sizes of capybaras from Argentina. Journal of Mammalogy. 2013;94(1):19-28.

8. Herrera EA. Capybara Social Behavior and Use of Space: Patterns and Processes. In: Moreira JR, Ferraz KMPMB, Herrera EA, Macdonald DW, eds. Capybara: Biology, Use and Conservation of an Exceptional Neotropical Species. New York: Springer Science Business Media; 2013. p. 195-207.

9. Oliveira JA, Bonvicino GR. Ordem Rodentia. In: Reis NR, Peracchi AL, Pedro WA, Lima IP. Mamíferos do Brasil. $2^{\mathrm{a}}$ ed. Londrina; 2011. p. 358-433.

10. Silva LFW. Criação de capivaras em cativeiro. São Paulo: Nobel. 1986. 
11. Gonzalez-Jimenez E. El capibara (Hydrochoerus hydrochaeris): estado actual de su producción. Roma: Organización de las Naciones Unidas para la Agricultura y la Alimentación (FAO);. 1995; 110 p.

12. Pinto GRM. Contagem de fezes como índice de abundância de capivaras (Hydrochaeris hydrochaeris) [dissertation]. Piracicaba: Universidade de São Paulo; 2003.

13. Verdade LM, Ferraz KMPMB. Capybaras in an anthropogenic habitat in southeastern Brazil. Brazilian Journal of Biology. 2006;66(1B):371-378.

14. Ferraz KMPMB, Ferraz SFB, Moreira JR, Couto HTZ, Verdade LM. Capybara (Hydrochoerus hydrochaeris) distribution in agroecosystems: a crossscale habitat analysis. Journal of Biogeography. 2007;34(2):223-230.

15. Ferraz KMPMB, Peterson AT, Scachetti-Pereira C, Vettorazzi CA, Verdade LM. Distribution Of Capybaras in an Agroecosystem, Southeastern Brazil, based on Ecological Niche Modeling. Journal of Mammalogy. 2009;90(1):189-194.

16. Galetti M, Eizirik E, Beisiegel B, Ferraz K, Cavalcanti S, Srbek-Araujo AC, et al. Atlantic rainforest's Jaguar in decline. Science. 2013;342(6161):930.

17. Pereira HFA, Eston MR. Biologia e Manejo de Capivaras (Hydrochoerus hydrochaeris) no Parque Estadual Alberto Löfgren, São Paulo, Brasil. Revista do Instituto Florestal. 2007;19(1):55-64.

18. Almeida AMR, Arzua M, Trindade PWS, Silva Junior A. Capivaras (Hydrochoerus hydrochaeris, Linnaeus, 1766) (Mammalia: Rodentia) em áreas verdes do município de Curitiba-(PR). Estudos de Biologia: Ambiente e Diversidade. 2013;35(84):9-16.

19. Almeida AMR, Biondi D. Área de uso de Hydrochoerus hydrochaeris L. em ambiente urbano. Ciência Animal Brasileira. 2014;15(3):369-376.

20. Moreira JR, Piovezan U. Conceitos de manejo de fauna, manejo de população problema eo exemplo da capivara. Documentos. Brasília: Embrapa Recursos Genéticos e Biotecnologia. 2005; 155 p.

21. Ferraz KMPMB, Lechevalier MA, Couto HTZ, Verdade LM. Damage caused by Capybaras in a Corn Field. Scientia Agricola. 2003;60(1):191-194.

22. Felix GA, Almeida-Paz ICL, Piovezan U, Garcia RG, Lima KAO, Nääs IA, et al. Feeding behavior and crop damage caused by capybaras (Hydrochoerus hydrochaeris) in an agricultural landscape. Brazilian Journal of Biology. 2014;74(4):779-786.

23. Bueno C, Faustino MT, Freitas SR. Influence of Landscape characteristics on Capybara road-kill on Highway BR-040, Southeastern Brazil. Oecologia Australis. 2013;17(2):320-327.

24. Huijser MP, Abra FD, Duffield JW. Mammal road mortality and cost-benefit analyses of mitigation measures aimed at reducing collisions with Capybara (Hydrochoerus hydrochaeris) in São Paulo State, Brazil. Oecologia Australis. 2013;17(1):129-146.

25. Moreira JR, Clarke JR, Macdonald DW. The testis of capybaras (Hydrochoerus hydrochaeris). Journal of Mammalogy. 1997;78(4):1096-1100.

26. Labruna MB, Kerber CE, Ferreira F, Faccini JLH, De Waal DT, Cennari SM. Risk factors to infections and their occurence on horses in the state of são Paulo, Brazil. Veterinary Parasitology. 2001;97(1):1-14.

27. Labruna MB, Whitworth T, Horta MC, Bouyer DH, McBride JW, Pinter A, et al. Rickettsia species infecting Amblyomma cooperi ticks from an area in the State of São Paulo, Brazil where, Brazilin spotted fever is endemic. Journal Clinical Microbiology. 2004;42(1):90-98.

28. Labruna MB. Ecology of Rickettsia in South America. Annals of the New York Academy of Sciences. 
2009;1166:156-166.

29. Souza CE, Moraes-Filho J, Ogrzewalska M, Uchoa FC, Horta MC, Souza SS, et al. Experimental infection of capybaras Hydrochoerus hydrochaeris by Rickettsia rickettsii and evaluation of the transmission of the infection to ticks Amblyomma cajennense. Veterinary Parasitology 2009;161(1-2):116-121.

30. Labruna MB. Brazilian SPOTTED FEVER: The Role of Capybaras. In: Moreira JR, Ferraz KMPMB, Herrera EA, Macdonald DW, eds. Capybara: Biology, Use and Conservation of an Exceptional Neotropical Species. New York: Springer Science Business Media; 2013.p. 371-383.

31. Perez CA, Almeida AF, Almeida A, Carvalho VHB, Balestrin DC, Guimarães MS, et al. Carrapatos do gênero Amblyomma (Acari: Ixodidae) e suas relações com os hospedeiros em área endêmica para febre maculosa no estado de São Paulo. Revista Brasileira de Parasitologia Veterinária. 2008;17(4):210-217.

32. Guedes E, Leite RC, Prata MCA, Pacheco RC, Walker DH, Labruna MB. Detection of Rickettsia rickettsii in the tick Amblyomma cajennense in a new Brazilian spotted fever-endemic area in the state of Minas Gerais. Memórias do Instituto Oswaldo Cruz. 2005;100(8):841-845.

33. Fortes FS, Santos LC, Cubas ZS, Barros-Filho IR, Biondo AW, Silveira I, et al. Anti-Rickettsia spp. antibodies in free-ranging and captive capybaras from southern Brazil. Pesquisa Veterinária Brasileira. 2011;31(11):1014-1018.

34. Brites-Neto J, Nieri-Bastos FA, Brasil J, Duarte KMR, Martins TF, Veríssimo CJ, et al. Environmental infestation and rickettsial infection in ticks in a Brazilian spotted fever-endemic area. Revista Brasileira de Parasitologia Veterinária. 2013;22(3):367-372.

35. Krawczak FS, Nieri-Bastos FA, Nunes FP, Soares JF, Moraes-Filho J, Labruna MB. Rickettsial infection in Amblyomma cajennense ticks and capybaras (Hydrochoerus hydrochaeris) in a Brazilian spotted feverendemic area. Parasites \& Vectors. 2014;7:7.

36. Nava S, Beati L, Labruna MB, Cáceres AG, Mangold AJ, Guglielmone AA. Reassessment of the taxonomic status of Amblyomma cajennense (Fabricius, 1787) with the description of three new species, Amblyomma tonelliae n. sp., Amblyomma interandinum n. sp. and Amblyomma patinoi n. sp., and reinstatement of Amblyomma mixtum, and Amblyomma sculptum (Ixodida: Ixodidae). Ticks and Tick-Borne Diseases. 2014;5(3):252-276.

37. Moreira JR. Capivaras: biologia, ecologia e controle. In: Meira AM, Cooper M, Ferraz KMPMB, Monti JA, Caramez RB, Delitti WBC, et al. Febre maculosa: dinâmica da doença, hospedeiros e vetores. Piracicaba: Universidade de São Paulo; 2013.

38. Pinter A. Febre maculosa brasileira - vigilância acarológica e controle. In: Meira AM, Cooper M, Ferraz KMPMB, Monti JA, Caramez RB, Delitti WBC. Febre maculosa: dinâmica da doença, hospedeiros e vetores. Piracicaba: Universidade de São Paulo; 2013.

39. Rego de Paula TA, Rodrigues MV. Manejo reprodutivo em grupos de capivaras (Hydrochoerus hydrochaeris). In: Meira AM, Cooper M, Ferraz KMPMB, Monti JA, Caramez RB, Delitti WBC, et al. Febre maculosa: dinâmica da doença, hospedeiros e vetores. Piracicaba: Universidade de São Paulo; 2013.

40. Labruna MB, Amaku M, Metzner JOA, Pinter A, Ferreira F. Larval Behavioral Diapause Regulates Life Cycle of Amblyomma cajennense (Acari: Ixodidae) in Southeast Brazil. Journal of Medical Entomology. 2003;40(2):170-178.

41. Labruna MB, Kasai N, Ferreira F, Faccini JL, Gennari SM. Seasonal dinamics of ticks (Acari: Ixodidae) on horses in the state of São Paulo, Brazil. Veterinary Parasitology. 2002;105(1):65-77.

42. Lemos ER, Alvarenga FB, Cintra ML, Ramos MC, Paddock CD, Ferebee TL, et al. Spotted fever in Brazil: a seroepidemiological study and description of clinical cases in an endemic area in the state of São Paulo. 
American Journal of Tropical Medicine and Hygiene. 2001;65(4):329-334.

43. São Paulo. Secretaria de Estado da Saúde de São Paulo. Centro de Vigilância Epidemiológica "Prof. Alexandre Vranjac". Febre maculosa brasileira. Suplemento Bepa. 2011;8(1).

44. Sponchiado J, Melo GL, Martins TF, Krawczak FS, Labruna MB, Cáceres NC. Association patterns of ticks (Acari: Ixodida: Ixodidae, Argasidae) of small mammals in Cerrado fragments, western Brazil. Experimental \& Applied Acarology. 2015;65(3):389-401.

45. Setzer J. A Distribuição normal das chuvas no Estado de São Paulo. Revista Brasileira de Geografia. 1946;8(1):3-70.

46. Setzer J. Atlas climático e ecológico do Estado de São Paulo. São Paulo: Comissão Interestadual da Bacia Paraná-Uruguai, Centrais Elétricas de São Paulo; 1966.

47. Alho CJR, Campos VM, Gonçalves HC. Ecologia de capivara (Hydrochoerus hydrochaeris, Rodentia) do Pantanal. I. Habitats, densidades e tamanho de grupo. Revista Brasileira de Biologia. 1987;47:88-97.

48. Yáber MC, Herrera EA. Vigilance, group size and social status in capybaras. Animal Behaviour. 1993;48(6):1301-1307.

49. Barreto GR, Herrera EA. Foraging patterns of capybaras in a seasonally flooded savanna of Venezuela. Journal of Tropical Ecology. 1998;14(1):87-98.

50. Ferraz KMPMB, Santos-Filho RM, Piffer TR, Verdade LM. Biologia e manejo da capivara: do controle de danos ao máximo de rendimento sustentável. In: Mattos WTRS, ed. A Produção Animal na Visão dos Brasileiros. Piracicaba: Sociedade Brasileira de Zootecnia. 2001; p. 580-588.

51. Conroy MJ, Noon BR. Mapping of species richness for conservation of biological diversity: conceptual and methodological issues. Ecological Applications. 1996;6(3):763-773.

52. Greenwood JJD, Gregory RD, Harris S, Morris PA, Yalden DW. Relations between abundance, body size and species number in British birds and mammals. Philosophical Transactions B.1996;351(1337):265278.

53. Bishir J, Lancia RA. On catch-effort methods of estimating animal abundance. Biometrics. 1996;52(4):1457-1466.

54. Cueto GR, Allekotte R, Kravetz FO. Scurvy in capybaras bred in captivity in Argentina. Journal of Wildlife Diseases. 2000;36(1):97-101.

55. Mohr CO. Table of equivalent populations of North American small mammals. The American Midland Naturalist. 1947;37(1):223-249.

56. Hayne DW. Calculation of size of home range. Journal of Mammalogy. 1949;30(1):1-18.

57. Jennrich RI, Turner FB. Measurement of non-circular home range. Journal of Theoretical Biology. 1969;22(2):227-237.

58. Otis DL, Burnham KP, White GC, Anderson DR. Statistical inference from capture data on closed animal populations. Wildlife Monographs. 1978;62:3-135.

59. White GC, Anderson DR, Burnham KP, Otis DL. Capture-recapture and removal methods for sampling closed populations. Los Alamos: Los Alamos National Laboratory; 1982.

60. Rexstad E, Burnham KP. User's guide for interactive program CAPTURE. Fort Collins: Colorado Cooperative Fish and Wildlife Research Unit; 1991.

61. Fernandez FAS. Métodos para estimativas de parâmetros populacionais por captura, marcação e recaptura.

Oecologia Brasiliensis. 1995;2(1):1-26. 
62. Terassini FA, Barbieri FS, Albuquerque S, Szabó MP, Camargo LM, Labruna MB. Comparison of two methods for collecting free-living ticks in the Amazonian forest. Ticks and Tick-Borne Diseases. 2010;1(4):194-196.

63. Flechtmann CHW. Ácaros de Importância Médico-Veterinária. $3^{a}$ ed. São Paulo: Editora Nobel. 1990; 192 p.

64. Barros-Battesti DM, Arzua M, Bechara GH. Carrapatos de importância médico-veterinária da região neotropical: um guia ilustrado para identificação de espécies. São Paulo: Vox/ICTTD-3/Instituto Butantan; 2006. 223 p.

65. Alho CJR, Rondon NL. Habitats, population densities and social structure of capybaras (Hydrochaeris hydrochaeris: Rodentia) in the Pantanal, Brasil. Revista Brasileira de Zoologia. 1987;4(2):139-149.

66. Alho CJR, Campos VM, Gonçalves HC. Ecologia de capivara (Hydrochoerus hydrochaeris, Rodentia) do Pantanal: II. Atividade, sazonalidade, uso do espaço e manejo. Revista Brasileira de Biologia. 1987;47:99110.

67. Borges LV, Colares IG. Feeding habits of capybaras (Hydrochoerus hydrochaeris, Linnaeus 1766), in the Ecological Reserve of Taim (ESEC - Taim) - south of Brazil. Brazilian Archives of Biology and Technology. 2007;50(3):409-416.

68. Vargas FC, Vargas SC, Moro MEG, Silva V, Carrer CRO. Monitoramento populacional de capivaras (Hydrochaeris hydrochaeris Linnaeus, 1766) em Pirassununga, SP, Brasil. Ciência Rural. 2007;37(4):11041108 .

69. Silva CF, Oliveira EF, Cunha EMF. Estrutura e dinâmica das populações de capivaras (Hydrochoerus hydrochaeris) em uma área aberta e em uma unidade de conservação: Perspectivas para o manejo. Revista em Agronegócios e Meio Ambiente. 2013;6(3):497-515.

70. Alho CJR, Campos VM, Gonçalves HC. Ecology, Social Behavior and Management of the capybara in the Pantanal of Brazil. In: Redford KH, Eisenber JF, eds. Advances in Neotropical Mammalogy. Gainesville: Sandhill Crane Press; 1989; p. 163-194.

71. Herrera EA. The behavioural ecology of capybara, Hydrochoerus hydrochaeris [dissertation]. Oxford: University of Oxford; 1986.

72. Salas V, Pannier E, Galíndez-Silva C, Gols-Ripoll A, Herrera EA. Methods for capturing and marking wild capybaras in Venezuela. Wildlife Society Bulletin. 2004;32(1):202-208.

73. Maher CR, Burger JR. Intraspecific variation in space use, group size, and mating systems of caviomorph rodents. Journal of Mammalogy. 2011;92(1):54-64.

74. Queirogas VL, Del-Claro K, Nascimento AR, Szabó MP. Capybaras and ticks in the urban areas of Uberlândia, Minas Gerais, Brazil: ecological aspects for the epidemiology of tick-borne diseases. Experimental \& Applied Acarology. 2012;57(1):75-82.

75. Silva Costa LF, Nunes PH, Soares JF, Labruna MB, Camargo-Mathias MI. Distribution of Rickettsia rickettsii in ovary cells of Rhipicephalus sanguineus (Latreille 1806) (Acari: Ixodidae). Parasites \& Vectors. 2011;4:222.

76. Gazeta GS, Souza ER, Abboud-Dutra AE, Amorim M, Barbosa PR, Almeida AB, et al. Potential vectors and hosts of ricettsia spp: epidemiological studies in the Vale do Paraíba, state of Rio de Janeiro/Brazil. Clinical Microbiology and Infection. 2009;15(s2):269-270.

77. Dantas-Torres F. Biology and ecology of the brown dog tick, Rhipicephalus sanguineus. Parasites \& Vectors. 2010;3:26. 
78. Estrada-Peña A, Guglielmone AA, Mangold AJ. The distribution and ecological 'preferences' of the tick Amblyomma cajennense (Acari: Ixodidae), an ectoparasite of humans and other mammals in the Americas. Annals of Tropical Medicine and Parasitology. 2004;98(3):283-292. 